\title{
Commentary: Iron deficiency of pregnancy - a new approach involving intravenous iron
}

Michael Auerbach ${ }^{1,2}$

From 2nd International Conference on Maternal and Newborn Health: Translating Research Evidence to Practice

Belagavi, India. 26-27 March 2018

\begin{abstract}
Iron deficiency anemia of pregnancy is common, especially in South Asia, and is associated with adverse maternal and fetal outcomes including increased incidences of maternal mortality, preterm labor and low birth weight. Screening for anemia alone is not sufficient to diagnose iron deficiency. Iron deficiency in neonates is associated with a statistically significant increment in cognitive and behavioral abnormalities which persist after iron repletion. Oral iron is the frontline standard but is associated with an unacceptably high incidence of gastrointestinal adverse events leading to poor adherence. Prospective evidence reports an incidence of neonatal iron deficiency up to 45\% even with oral iron supplementation. New evidence reports oral iron ingestion increases serum hepcidin leading to decreased absorption suggesting further decreasing efficacy. Published evidence reports that intravenous iron is safe and effective in the second and third trimesters of pregnancy. Intravenous iron is the preferred route when there is oral iron intolerance or in those situations where oral iron is ineffective or harmful. Intravenous iron is also preferred if the anemia is severe $(<$ $8 \mathrm{~g} / \mathrm{dL}$ ) in the second trimester or at any time in the third trimester when there is little expectation that adequate quantities of iron will be delivered to the fetus as iron requirements increase in each trimester. Guidelines for maternal and neonatal screening and treatment lack consistency and differ between the United States and Europe. New formulations of intravenous iron with complex carbohydrate cores that bind elemental iron more tightly mitigating the release of large quantities of labile free iron allow the administration of complete replacement doses in 15-60 min. The preponderance of published evidence suggests that intravenous iron is underutilized in pregnancy and guidelines suggesting there is insufficient evidence to recommend the routine screening and treatment of iron deficiency in gravidas should be revisited. The major recommendation from this commentary is that in low-income countries, a trial or demonstration project to test the efficacy, safety, cost and feasibility of the administration of intravenous iron to anemic and/or iron-deficient women be undertaken.
\end{abstract}

Keywords: Iron deficiency, Anemia of pregnancy, Intravenous Iron, Neonatal Iron deficiency

\section{Background}

The estimated incidence of anemia of pregnancy varies between eight and $20 \%$, largely dependent on the economic status of the measured populations [1-4] with some areas of India and Pakistan reporting up to $90 \%$. In some of these areas, hemoglobin levels of $8 \mathrm{mg} / \mathrm{dl}$ are found in nearly $10 \%$ of the pregnant population despite the availability and use of oral iron and vitamin preparations (unpublished data). While often considered of

Correspondence: mauerbachmd@abhemonc.com

${ }^{1}$ Auerbach Hematology and Oncology, Baltimore, MD, USA

${ }^{2}$ Georgetown University, Washington DC, USA minor clinical significance, iron deficiency is associated with significant adverse maternal and fetal outcomes during pregnancy with a reported two-fold increased incidence of preterm labor and a three-fold increase in the incidence of low birth weight [5]. Screening for anemia alone is not sufficient to diagnose iron deficiency. If iron deficiency and heavy vaginal bleeding are present at the beginning of pregnancy, the incidence of preterm labor is increased five-fold [2]. Iron deficient mothers, irrespective of anemia, are at risk of delivering iron deficient neonates [6-8]. Recent data suggest that iron deficient neonates exhibit delayed growth and development as

(c) The Author(s). 2018 Open Access This article is distributed under the terms of the Creative Commons Attribution 4.0 International License (http://creativecommons.org/licenses/by/4.0/), which permits unrestricted use, distribution, and reproduction in any medium, provided you give appropriate credit to the original author(s) and the source, provide a link to the Creative Commons license, and indicate if changes were made. The Creative Commons Public Domain Dedication waiver (http://creativecommons.org/publicdomain/zero/1.0/) applies to the data made available in this article, unless otherwise stated. 
well as a statistically significant increment of cognitive and behavioral abnormalities which persist even after iron repletion [9]. Oral iron therapy, the current frontline standard, is often not optimal for iron deficiency in pregnancy. Intravenous iron is safe, effective and should be considered early in the treatment paradigm for iron deficient gravidas, irrespective of the presence or absence of anemia.

The Centers for Disease Control (CDC), American College of Obstetricians and Gynecologists (ACOG) and the United States Preventive Service Task Force (USPSTF) all recommend routine screening for anemia during pregnancy and the CDC and ACOG recommend low-dose iron supplementation for all pregnant women $[5,10,11]$. In all of these guidelines there remains no admonition to screen for iron deficiency at the beginning of pregnancy or at any time thereafter in the absence of anemia. These recommendations were supported by a recent USPSTF publication which reported that "there is insufficient evidence that routine prenatal screening and supplementation for iron deficiency anemia improves maternal or infant clinical health outcomes, but supplementation may improve maternal hematologic indices" [12]. While these recommendations are syntactically accurate due to the lack of published outcome data, a plethora of published evidence suggests the recommendations be revisited. This position is supported by The Cochrane Collaboration, reporting that despite the high incidence and burden of disease associated with iron deficiency anemia of pregnancy, there "is a paucity of quality trials assessing clinical maternal and neonatal effects of iron administration in pregnant women with anemia [4]. That being said, while prospective studies may be absent for proving that routine screening and supplementation is beneficial, ample data exist to recommend revisiting the USPSTF conclusions. In 1967 Scott and Pritchard reported that $58 \%$ of healthy 18 year old, non-pregnant, women have absent hemosiderin on marrow aspiration, suggesting low iron stores [13]. The current USPSTF recommendations will subsequently miss all those with iron deficiency without anemia. Pregnant women presenting to their obstetricians are not screened for iron deficiency unless anemic with a low hemoglobin concentration. Clinicians may miss up to $55 \%$ of iron deficient gravidas with significantly abnormal values when iron parameters are not added to the screening laboratory tests [14]. Iron deficiency in pregnancy precedes anemia. Further, multiple studies report significant morbidity from iron deficiency, which may occur in the absence of anemia. Examples of sequelae in the fetus include neonatal and childhood brain growth and developmental abnormalities with adverse effects on myelination, neurotransmitters and brain programming [15]. Additionally, a two-fold increased incidence of preterm birth, a three-fold increase in low birth weight, and small for gestational age infants have been reported [5]. Published evidence indicates that low serum maternal ferritin concentrations are associated with iron deficiency in neonates [16] and associated with a statistically significant increment in both cognitive and behavioral abnormalities which appears to be long lasting and detectable up to 19 years of age $[9,17]$. These conclusions are supported by evidence that in 6-24 month old infants with iron deficiency anemia there is increased risk for poorer cognitive, motor, social-emotional and neurophysiologic development [18]. In addition to negative effects on the fetus, maternal iron deficiency anemia is associated with increased risk for caesarean section, increases in transfusion, perinatal bleeding, pre-eclampsia, placental abruption, abnormal maternal thyroid status, impaired wound healing, cardiac failure and death [19-21].

\section{Iron repletion}

Iron repletion has been shown to decrease morbidity in those with heavy uterine bleeding, inflammatory bowel disease, chronic kidney disease, cancer and chemotherapy induced anemia, heart failure, hereditary hemorrhagic telangiectasia, bariatric surgery, the pre-, peri- and postoperative periods and in critically ill patients. It is therefore reasonable to infer that in the absence of harm, a similar benefit to iron deficient gravidas would be observed leading to the credible conclusion that, given the absence of quality prospective outcomes data, we should err on the side of repletion until such data are available. This conclusion is additionally supported by a study of 2400 urban Chinese women which reported that up to $45 \%$ of infants were iron deficient despite oral supplementation [6]. Iron requirements dramatically increase in pregnancy to accommodate an expanding red cell volume, growing fetus and placenta plus any anticipated or unanticipated blood loss at delivery. This is of special significance if a caesarian section is required. Published evidence states that daily iron requirements increase from $0.8,4-5$ to $6 \mathrm{mg} /$ day in the first second and third trimesters respectively [7] (Fig. 1). It is estimated that Iron requirements in pregnancy may exceed $1000 \mathrm{mg}$, with $500 \mathrm{mg}$ required for red cell expansion, $300-350 \mathrm{mg}$ for the developing fetus and placenta, with variable losses at delivery [8].

The World Health Organization estimates that the worldwide incidence of anemia of pregnancy approaches $50 \%$ [19]. It defines anemia of pregnancy as a hemoglobin level of less than $11 \mathrm{~g} / \mathrm{dL}$, or hematocrit < $33 \%$, at any time during pregnancy with the CDC defining anemia of pregnancy as $\mathrm{Hb}<11 \mathrm{~g} / \mathrm{dL}$, or hematocrit $<33 \%$ during the first and third trimesters and less than $10.5 \mathrm{~g} / \mathrm{dL}$, or a hematocrit $<32 \%$ in the second trimester $[5,22]$.

The current standard for iron deficiency is oral iron administered as two to three $325 \mathrm{mg}$ tablets containing approximately 50-65 mg elemental iron daily. Gastric 
Q. ASH' Consultative Hematology Course

Daily Iron Requirement in Pregnancy

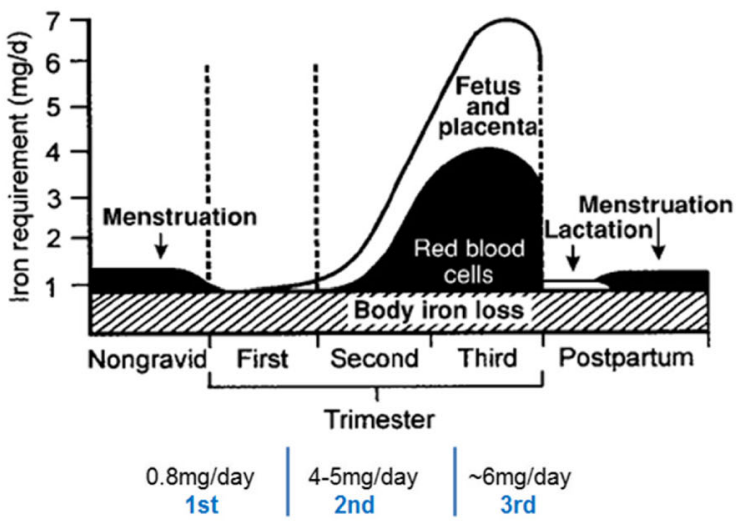

Bothwell. Am J Clin Nutr 2000;72(suppl):2575-64S

Fig. 1 Iron requirements throughout pregnancy

\begin{tabular}{|c|c|c|c|}
\hline \multicolumn{2}{|l|}{$\begin{array}{l}\text { Study } \\
\text { ID }\end{array}$} & \multirow[t]{2}{*}{ OR $(95 \% \mathrm{Cl})$} & \multirow[t]{2}{*}{$\begin{array}{l}\% \\
\text { Weight }\end{array}$} \\
\hline Other & & & \\
\hline Aganwal et al, 2006 & & $0.60(0.22,1.58)$ & 6.98 \\
\hline Auerbach et al, 2004 & & $5.54(0.22,139.00)$ & 1.28 \\
\hline Bhandal and Russell, 2006 & & $23.28(1.23,439.32)$ & 1.51 \\
\hline Breymann et al, 2008 & & $3.13(1.24,7.88)$ & 7.32 \\
\hline Charytan et al, 2005 & & $3.84(1.36,10.86)$ & 6.59 \\
\hline Guerra Merino, 2012 & & $5.91(0.23,151.15)$ & 1.27 \\
\hline Henry et al, 2007 & & $1.05(0.51,2.17)$ & 8.76 \\
\hline Mudge et al, 2012 & & $2.13(0.50,9.04)$ & 4.56 \\
\hline Seid et al, 2008 & & $5.66(1.61,19.87)$ & 5.40 \\
\hline Strickland et al, 1977 & & $5.54(0.25,123.08)$ & 1.38 \\
\hline Tokars et al, 2010 & & $1.65(0.61,4.47)$ & 6.86 \\
\hline Van Wyck et al, 2005 & & $2.21(0.90 .5 .47)$ & 7.46 \\
\hline Van Wyck et al, 2007 & & $4.72(2.34,9.51)$ & 8.93 \\
\hline Van Wyck et al, 2009 & & $4.58(2.06,10.17)$ & 8.20 \\
\hline Subtotal $(1-$ squared $=48.8 \%, p=0.020)$ & & $2.63(1.70,4.06)$ & 76.50 \\
\hline \multicolumn{4}{|l|}{ Pregnant } \\
\hline Al-Momen et al, 1996 & & $46.81(2.74,799.83)$ & 1.61 \\
\hline Bayoumeu et al, 2002 & & $3.12(0.12,80.39)$ & 1.27 \\
\hline Bencaiova et al, 2009 & & $57.06(3.43,950.30)$ & 1.63 \\
\hline Kochhar et al, 2013 & $\rightarrow-1$ & $2.09(0.36,11.95)$ & 3.52 \\
\hline Vazquez Pacheco, J. 1980 & & $11.18(0.56,222.98)$ & 1.47 \\
\hline Subtotal $(1-$ squared $=33.4 \%, p=0.199)$ & $>$ & $9.44(2.23,39.93)$ & 9.49 \\
\hline \multicolumn{4}{|l|}{ IBD } \\
\hline Kulnigg et al, 2008 & & $2.25(0.55,9.32)$ & 4.66 \\
\hline Lindgren et al, 2009 & & $29.48(1.68,517.45)$ & 1.58 \\
\hline Reinisch et al, 2013 & & $2.79(0.61,12.71)$ & 4.28 \\
\hline Schroder et al, 2005 & $\longrightarrow$ & $2.63(0.45,15.23)$ & 3.49 \\
\hline Subtotal $(1-$ squared $=0.0 \%, p=0.454$ ) & & $3.14(1.34,7.36)$ & 14.01 \\
\hline Overall $(1-$ squared $=41.6 \%, p=0.020)$ & $\diamond$ & $3.05(2.07,4.48)$ & 100.00 \\
\hline \multicolumn{4}{|l|}{ NOTE: Weights are from random effects analysis } \\
\hline $\begin{array}{r}1 \\
.00105\end{array}$ & & & \\
\hline
\end{tabular}

Fig. 2 Effect of daily ferrous sulfate supplementation on the incidence of gastrointestinal side-effects in intravenous iron-controlled randomized control trials. With Permission: Tolkien et al. [23] 
acid is necessary to conjugate the iron to amino acids, sugars and vitamin $C$ which protects the elemental iron from conversion to ferric hydroxide in the proximal duodenum. This would be unabsorbable as a result of the massive alkaline secretions of the pancreas necessary for normal absorption. Parenthetically, this information mitigates use in gravidas who have undergone bariatric surgery with either roux-en-Y or biliopancreatic bypass procedures. A variety of other formulations such as heme polypeptide, enteric coated and timed release iron, ostensibly designed to increase tolerability, have been compared to ferrous sulfate with no improvement in toxicity and with equivalent efficacy.

Oral iron is inexpensive, readily available, and easy to obtain. However, more than $70 \%$ of those to whom it is prescribed, complain of significant gastrointestinal perturbation which includes metallic taste, gastric irritation, and worsening constipation resulting in poor adherence [23] (Fig. 2). These symptoms are especially onerous as constipation is rife in pregnancy due to the rapidly increasing progesterone levels which slow bowel transit, and the enlarging uterus pressing posteriorly on the rectum. Further complicating the use of oral iron is recently published evidence demonstrating increments in serum hepcidin levels for approximately $48 \mathrm{~h}$ after ingestion of an iron tablet (which impair iron absorption and release) [24]. Hepcidin, the hepatic synthesized iron regulatory protein, decreases iron absorption at the level of the intestinal epithelium and release from iron laden circulating macrophages. Recently published supporting evidence using radiolabeled oral ferrous sulfate reported improved absorption with a single tablet on alternate days compared to use of daily or twice daily ferrous sulfate [25]. While this gradual repletion may suffice in an individual who is not pregnant, the need in pregnancy is more urgent. It is not credible to expect rapid, clinically meaningful iron repletion, essential for the developing fetus, with a small dose of oral iron.

Lending further support to the inadequacy of oral iron in many gravid women is published evidence reporting that a ferritin level less than $15 \mathrm{ng} / \mathrm{ml}$ in the mother compromises iron status of the growing fetus whose iron requirements for normal brain development are maximal from week 34 on [6]. Current guidelines do not recommend routine screening for iron deficiency in newborns. Yet, current guidelines also do not recommend routine prenatal screening and supplementation for iron deficiency in the absence of anemia [12]. These issues of concern question the current paradigm of frontline oral iron therapy in moderately to severely anemic pregnant women after the first trimester and suggest that the parenteral route may be a preferred option.

The use of intravenous iron in pregnancy remains sporadic at best. Whereas intravenous iron has been shown to be nearly uniformly safe and effective, with serious adverse events extremely rare, numerous published trials, irrespective of which of the currently available intravenous iron formulations is used, recommendations for its use vary greatly. The 2008 ACOG Practice Bulletin recommends intravenous iron in the "rare patient who cannot tolerate or will not take modest doses of oral iron" with the caveat that patients with severe malabsorption may benefit from parenteral iron [11]. In contradistinction, the 2012 United Kingdom guidelines states that "parenteral iron should be considered from the second trimester onwards and during the postpartum period for women with confirmed iron deficiency who fail to respond to or are intolerant of oral iron" [26]. And, in a recent review of the treatment of anemia in pregnancy, Achebe and Gafter-Gvili recommend intravenous iron for oral iron intolerant 2nd and 3rd trimester patients, 2nd trimester gravidas with hemoglobin concentrations less than $10.5 \mathrm{~g} / \mathrm{dL}$ and in all in the third trimester with iron deficiency anemia. India, has no country-wide recommendations related to intravenous iron use, although some of the states, including Karnataka, where this conference took place, recommend its use in certain circumstances. There are no existing guidelines for the treatment of nonanemic, iron deficient pregnant women [22].

The successful use of intravenous iron in pregnancy is hardly new. In 1964 and in 1973, two different studies of intravenous iron in iron deficient gravidas reported the safety and efficacy in more than 2500 pregnant women, of complete replacement dosing with iron dextran in a single setting $[27,28]$. Nearly ubiquitous efficacy was observed without any serious adverse events. Yet over 50 years later, the first United States prospective study of intravenous iron in pregnancy was recently published [21]. Seventy-four oral iron intolerant, second and third trimester iron deficient gravidas were questioned for oral iron intolerance, and if present, were treated with intravenous iron. All received $1000 \mathrm{mg}$ of low-molecular weight iron dextran in $250 \mathrm{ml}$ normal saline over $1 \mathrm{~h}$ without premedication, unless multiple drug allergies or asthma was present. In this case, methylprednisolone was administered prior to the test dose. Fifteen minutes after a test dose, the remainder was infused over the balance of $1 \mathrm{~h}$ and no serious adverse events were observed. Those enrolled were called at one, two and 7 days to assess for delayed reactions. Four weeks post-infusion or postpartum, hemoglobin concentrations and iron parameters were measured. Fifty-eight of 73 women were questioned about interval growth and development of their babies. The mean pre- and post-hemoglobin concentrations were 9.7 and $10.8 \mathrm{~g} / \mathrm{dL}(P<0.00001)$ and ferritin of 14.5 and $126.3 \mathrm{ng} / \mathrm{mL}(P<0.000001)$ respectively. While six patients experienced transient minor infusion reactions, all of which resolved without therapy, there were no 
serious adverse events observed. Data for 58 infants, ages 3 months to 3 years, were available. One had delayed development reported which resolved by 11 months, the remaining 57 were reported as normal. None were diagnosed with iron deficiency. The authors concluded that intravenous iron has less toxicity and, consistent with the preponderance of published evidence, is more effective than oral iron, supporting moving its use closer to frontline therapy.

These data support a previously published observational study by the same group of 189 consecutive, nonselected, oral iron intolerant second and third trimester gravidas who received $1000 \mathrm{mg}$ of low molecular weight iron dextran in $1 \mathrm{~h}$ [29]. Hemoglobin concentrations increased by $1-1.9 \mathrm{~g} / \mathrm{dL}$ in $58 \%$ and by greater than $2 \mathrm{~g}$ in $24 \%$. Anemia resolved in $95 \%$. No serious adverse events were observed. The authors concluded that a large, single, rapidly administered dose of intravenous low-molecular weight iron dextran was effective, safe and convenient. A recent prospective international, open-label randomized controlled study compared ferric carboxymaltose to oral iron. More study participants achieved correction with the intravenous formulation without the frequent gastrointestinal side effects seen in the oral iron arm [30]. In yet another prospective comparison of low-molecular weight iron dextran and ferric carboxymaltose, the authors reported a hemoglobin rise of $2.34-2.57 \mathrm{~g} / \mathrm{dL}$ at 4 weeks without any serious adverse events in either group [31] and concluded that both formulations are effective and safe, with low risk of adverse events.

It is not the purview of this review to provide an exhaustive compendium of the litany of published evidence on intravenous iron in pregnancy. However, the illustrative examples provided are consistent with the overwhelming preponderance of published data supportive of the safety and efficacy of all intravenous iron formulations in correcting iron deficiency in gravidas. A consistent finding in virtually all published evidence is the absence of serious adverse events. Why then is there such resistance to incorporate the early use of intravenous iron into the treatment paradigm of iron deficiency in pregnancy among obstetricians and gynecologists? The answer may be that the folklore of fear of serious adverse events, which include anaphylaxis, coupled with the fact that no intravenous iron formulation has been assigned the highest safety rating from FDA discourages obstetricians already struggling in a litigious environment (particularly in the United States). Misinterpretation of minor infusion reactions as a serious hypersensitivity further mitigates its use [32]. Older formulations of intravenous iron consisting of high molecular weight iron dextran, which are no longer available, were associated with an incidence of severe hypersensitivity of 1-3\% [33]. While iron sucrose and ferric gluconate are safe and effective, their smaller carbohydrate cores bind elemental iron less tightly and preclude administration of doses larger than 200$300 \mathrm{mg}$ in a single setting due to the release of increased amounts of labile free iron [34]. Newer formulations in the United States, Europe and Asia, which include low molecular weight iron dextran, ferric carboxymaltose, ferumoxytol, and iron isomaltoside are able to be administered as a complete replacement dose in a short single visit of 15-60 min as a result of complex carbohydrate cores which bind the elemental iron more tightly, limiting the amount of labile free iron. These formulations are associated with a lower incidence of infusion reactions than either iron sucrose or ferric gluconate and have a much lower incidence of serious adverse events, with an estimated incidence of such serious events of less than 1 : 250,000 doses [35].

These composite conclusions parallel results from a large meta-analysis of 103 trials comprising over 10,391 patients who were treated with intravenous iron compared to 4044 given oral iron, 1329 no iron, 3335 placebo and 155 treated with intramuscular iron [36]. In this large population, 935 women were pregnant and another 748 peripartum. Parenthetically, intramuscular iron is painful, requires multiple injections, stains the buttock and has been associated with gluteal sarcomas and subsequently should be avoided. Overall, while uncommon, infusion reactions were observed with intravenous iron although there was no increase in serious adverse events with intravenous iron compared to controls, including placebo (95\% CI 0 . 93-1.17, 97 trial $\mathrm{I}^{2}=9 \%$ ). No difference in safety or efficacy was reported among any of the studied formulations, consistent with all prospective, intra-institutional retrospective studies and meta-analyses [37].

\section{Conclusions}

While initial cost of intravenous iron formulations for under resourced countries may be problematic, clinical trials to determine cost effectiveness, and public pricing availability may mitigate these concerns. Therefore, the information in this commentary recommends a revisiting of the current international guidelines for the screening and treatment of anemia in pregnancy. Thus it is suggested:

1. All newly diagnosed gravidas, irrespective of hemoglobin level at presentation to their obstetricians, midwives or other providers, be screened for iron deficiency to include serum iron, total iron binding capacity, percent transferrin saturation and serum ferritin.

2. If iron deficiency is present in the first trimester one ferrous sulfate tablet every other day should be taken. We acknowledge these recommendations may not be practical for much of the world's pregnant woman with limited health care budgets, 
however the potential improved outcomes may prove cost effective.

3. If iron deficiency is diagnosed in the second trimester, the hemoglobin is greater than $8 \mathrm{~g} / \mathrm{dL}$ and the mother's serum ferritin is greater than $15 \mathrm{ng} / \mathrm{ml}$, one ferrous sulfate tablet every other day should be taken with a rapid switch to intravenous iron if the therapy proves ineffective or is poorly tolerated. If the hemoglobin level is less than $8 \mathrm{~g} / \mathrm{dL}$ or the mother's serum ferritin is less than $15 \mathrm{ng} / \mathrm{ml}$, the intravenous route is preferable.

4. Intravenous iron is the preferred route of replacement if required, in the third trimester.

5. Neonates at risk for iron deficiency should be screened at birth. These include preterm infants, infants of diabetic mothers, infants born to anemic or iron deficient mothers, those with parasitic infestation or malaria, HIV, and those who had chronic hypoxia in utero (infants of smokers).

6. In low income countries, a trial or demonstration project to test efficacy, safety, cost and feasibility of the routine administration of intravenous iron appears prudent.

7. A prospective comparison of oral to intravenous iron with screening of neonates appears prudent.

\section{Funding}

Publication charges for this supplement were funded by the University of British Columbia PRE-EMPT (Pre-eclampsia/Eclampsia, Monitoring, Prevention and Treatment) initiative supported by the Bill \& Melinda Gates Foundation.

\section{Author's contributions}

The author read and approved the final manuscript.

\begin{abstract}
About this supplement
This article has been published as part of Reproductive Health Volume 15 Supplement 1, 2018: Improving pregnancy outcomes - Proceedings of the 2nd International Conference on Maternal and Newborn Health: Translating Research Evidence to Practice. The full contents of the supplement will be available online at https://reproductive-health-journal.biomedcentral.com/ articles/supplements/volume-15-supplement-1.
\end{abstract}

\section{Ethics approval and consent to participate}

Not applicable.

\section{Competing interests}

The author declares that he has no competing interests.

\section{Publisher's Note}

Springer Nature remains neutral with regard to jurisdictional claims in published maps and institutional affiliations.

Published: 22 June 2018

\section{References}

1. Milman N. Anemia-still a major health problem in many part of the world! Ann Hematol. 2011;90:369-77.

2. Scholl T, Hediger M, Fischer $R$, et al. Anemia vs iron deficiency: increased risk of preterm delivery in a prospective study. Am J Clin Nutr. 1992;55:985-8.

3. Stevens $G$, Finucane $M$, De-Regil $L$, et al. Global, regional and national trends in haemoglobin concentration and prevalence of total and severe anaemia in children and pregnant and non-pregnant women for 1995-
2011: a systematic analysis of population-representative data. Lancet Glob Health. 2013;1:e16-25.

4. Reveiz L, Gyte G, Cuervo L, et al. Treatments for iron-deficiency anemia in pregnancy (review). Cochrane Database Syst Rev. 2011;10:CD003094. [PMID:2195735]

5. Recommendations to prevent and control iron deficiency in the United States. Centers for Disease Control and Prevention. MMWR Recomm Rep. 1998;47(RR-3):1-29.

6. Zhao G, Xu G, Zhou M, et al. Prenatal iron supplementation reduces maternal anemia, iron deficiency, and iron deficiency anemia in a randomized clinical trial in China, but iron deficiency remains widespread in mothers and neonates. J Nutr. 2015;145:1916-23.

7. Bothwell TH. Iron requirements in pregnancy and strategies to meet them. Am J Clin Nutr. 2000;72(suppl 1):257S-264.

8. Scholl T. Maternal iron status: relation to fetal growth, length of gestation, and iron endowment of the neonate. Nutr Rev. 2011;69(Supp 1):S23-9.

9. Congdon $\mathrm{E}$, Westerlung $\mathrm{A}$, Algarin $\mathrm{C}$, et al. Iron deficiency in infancy is associated with altered neural correlates of recognition memory at 10 years. J Peds. 2012:160:1027-3.

10. U.S. Preventive Services Task Force. Screening for iron deficiency anemia_including iron prophylaxis. In: Guide to clinical preventive services. 2nd ed. Alexandria: International Medical Publishing; 1996. p. 231-46.

11. American College of Obstetricians and Gynecologists. ACOG practice bulletin no. 95: anemia in pregnancy. Obstet Gynecol. 2008;1112:201-7.

12. Cantor A, Bougatsos C, Dana T. Routine iron supplementation and screening for iron deficiency anemia in pregnancy: a systematic review for the U.S. preventive services task force. Ann Intern Med. 2015;162:566-76.

13. Scott D, Pritchard J. Iron deficiency in healthy young college women. JAMA 1967:199:147-50.

14. Johnson S, Lang A, Sturm M, et al. Iron deficiency without anemia: a common yet under-recognized diagnosis in young women with heavy menstrual bleeding. J Pediatr Adolesc Gynecol. 2016;29:628-31.

15. Roncagliolo M, Garrido M, Walter T, et al. Evidence of altered central nervous system development in infants with iron deficiency anemia at 6 mo: delayed maturation of auditory brainstem responses. Am J Clin Nutr. 1998:68:683-90.

16. Shao J, Lou J, Rao R, et al. Maternal serum ferritin concentration is positively associated with newborn iron stores in women with low ferritin status in late pregnancy. J Nutr. 2012;142:2004-9.

17. Lozoff B, Jimenez E, Smith J. Double burden of iron deficiency in infancy and low socioeconomic status: a longitudinal analysis of cognitive test scores to age 19 years. Arch Pediatr Adolesc Med. 2006;160:1108-13.

18. Lozoff B. Iron deficiency and child development. Food Nutr Bull. 2007;28: S560-71.

19. World Health Organization. Iron deficiency Anaemia: assessment, prevention and control: a guide for Programme manager. Geneva: World Health Organization; 2001.

20. Drukker $L$, Hants $Y$, Farkash $R$, et al. Iron deficiency anemia at admission for labor and delivery is associated with an increased risk for caesarean section and adverse maternal and neonatal outcomes. Transfus. 2015;55:2799-806.

21. Auerbach M, James S, Nicoletti M, et al. Results of the first American prospective study of intravenous iron in oral iron-intolerant iron-deficient gravidas. Am J Med. 2017:130:1402-7.

22. Achebe M, Gafter-Gvili A. How I treat anemia in pregnancy: iron, cobalamin, and folate. Blood. 2017:129:940-9.

23. Tolkien Z, Stecher L, Mander A, et al. Ferrous sulfate supplementation causes significant gastrointestinal side-effects in adults: a systematic review and meta-analysis. PLoS One. 2015;10:e0117383.

24. Moretti D, Goede J, Zeder C, et al. Oral iron supplements increase hepcidin and decrease iron absorption from daily or twice-daily doses in iron depleted young women. Blood. 2015;126:1981-9.

25. Stoffel N, Cercamondi C, Brittenham G, et al. Iron absorption from oral iron supplements given on consecutive versus alternate days and as single morning doses versus twice-daily split dosing in iron-depleted women: two open-label, randomized controlled trials. Lancet Haematol. 2017;4:e524-33.

26. Pavord S, Myers B, Robinson S, et al. British Committee for Standard in Haematology: UK guidelines on the management of iron deficiency in pregnancy. Br J Haematol. 2012;156:588-600.

27. Varde K. Treatment of 300 cases of iron deficiency of pregnancy by total dose infusion of iron-dextran complex. J Obstat Gynaecol Br Comm. 1964; 71:919-22. 
28. Kanakaraddi V, Hoskatti C, Nadig V, et al. Comparative therapeutic study of T.D.I. And I.M. Injections of iron dextran complex in anaemia. J Assoc Phys India. 1973;21:849-53.

29. Wong L, Smith S, Gilstrop M, et al. Safety and efficacy of rapid $1,000 \mathrm{mg}$ in $1 \mathrm{hr}$ ) intravenous iron dextran for treatment of maternal iron deficient anemia of pregnancy. Am J Hematol. 2016;91:590-3.

30. Breymann C, Milman N, Mezzacasa A, et al. Ferric carboxymaltose vs oral iron in the treatment of pregnant women with iron deficiency anemia: an international, open-label, randomized controlled trial (FER-ASAP). J Perinat Med. 2017:45:443-53.

31. Myers B, Myers O, Moore J. Comparative efficacy and safety of intravenous ferric carboxymaltose (Ferinject) and iron (III) hydroxide dextran (Cosmofer) in pregnancy. Obstet Med. 2012;5:105-7.

32. Auerbach M, Ballard H, Glaspy J. Clinical update: intravenous iron for anaemia. Lancet. 2007:369:1502-4.

33. Auerbach $\mathrm{M}$, Ballard $\mathrm{H}$. Clinical use of intravenous iron: administration, efficacy and safety. Hematol Am Soc Hematol Educ Program. 2010;2010:338-47.

34. Chandler G, Hachowal J, Macdouall I. Intravenous iron sucrose: establishing a safe dose. Am J Kidney Dis. 2001;38:988-91.

35. Chertow G, Mason P, Vaage-Milsen $\mathrm{O}$, et al. Update on adverse drug events associated with parenteral iron. Nephrol Dial Transplant. 2006;21:378-82.

36. Avni T, Beber A, Grossman A, et al. The safety of intravenous iron preparations: systematic review and meta-analysis. Mayo Clin Proc. 2015; 90:12-23.

37. Auerbach $M$, Macdougall I. The available intravenous iron formulatons: history, efficacy and toxicology. Hemodial Int. 2017;21(Suppl1):S83-92.

Ready to submit your research? Choose BMC and benefit from:

- fast, convenient online submission

- thorough peer review by experienced researchers in your field

- rapid publication on acceptance

- support for research data, including large and complex data types

- gold Open Access which fosters wider collaboration and increased citations

- maximum visibility for your research: over $100 \mathrm{M}$ website views per year

At BMC, research is always in progress.

Learn more biomedcentral.com/submissions 